\title{
Thin $\mathrm{SnO}_{\mathbf{x}}$ films for surface plasmon resonance enhanced ellipsometric gas sensing (SPREE)
}

\author{
Daniel Fischer ${ }^{1}$, Andreas Hertwig ${ }^{*}$, Uwe Beck ${ }^{1}$, Volkmar Lohse ${ }^{2}$, Detlef Negendank ${ }^{2}$, \\ Martin Kormunda ${ }^{3}$ and Norbert Esser ${ }^{4}$
}

Open Access

\author{
Full Research Paper \\ Address: \\ ${ }^{1}$ BAM, Division 6.7, Surface Modification and Measurement \\ Technique, Unter den Eichen 44-46, 12203 Berlin, Germany, ${ }^{2}$ BAM, \\ Division 2.1, Gases, Gasplants, Unter den Eichen 44-46, 12203 \\ Berlin, Germany, ${ }^{3}$ J.E.Purkinje University, Faculty of Science, \\ Department of Physics, Ceske mladeze 8, 40096 Usti nad Labem, \\ Czech Republic and ${ }^{4}$ Leibniz Institut für Analytische Wissenschaften \\ ISAS e.V., Schwarzschildstr. 12, 12489 Berlin, Germany \\ Email: \\ Andreas Hertwig ${ }^{*}$ - andreas.hertwig@bam.de \\ * Corresponding author \\ Keywords: \\ doped tin oxide; ellipsometry; gas sensing; surface plasmon \\ resonance; thin films; transparent conductive oxides
}

Beilstein J. Nanotechnol. 2017, 8, 522-529.

doi:10.3762/bjnano.8.56

Received: 01 July 2016

Accepted: 16 February 2017

Published: 28 February 2017

This article is part of the Thematic Series "Functional materials for environmental sensors and energy systems".

Guest Editor: M. Penza

(C) 2017 Fischer et al.; licensee Beilstein-Institut.

License and terms: see end of document.

\begin{abstract}
Background: Gas sensors are very important in several fields like gas monitoring, safety and environmental applications. In this approach, a new gas sensing concept is investigated which combines the powerful adsorption probability of metal oxide conductive sensors (MOS) with an optical ellipsometric readout. This concept shows promising results to solve the problems of cross sensitivity of the MOS concept.
\end{abstract}

Results: Undoped tin oxide $\left(\mathrm{SnO}_{\mathrm{x}}\right)$ and iron doped tin oxide $\left(\mathrm{Fe}: \mathrm{SnO}_{\mathrm{x}}\right)$ thin add-on films were prepared by magnetron sputtering on the top of the actual surface plasmon resonance (SPR) sensing gold layer. The films were tested for their sensitivity to several gas species in the surface plasmon resonance enhanced (SPREE) gas measurement. It was found that the undoped tin oxide $\left(\mathrm{SnO} \mathrm{O}_{\mathrm{x}}\right)$ shows higher sensitivities to propane $\left(\mathrm{C}_{3} \mathrm{H}_{8}\right)$ then to carbon monoxide (CO). By using $\mathrm{Fe}: \mathrm{SnO}_{\mathrm{x}}$, this relation is inverted. This behavior was explained by a change of the amount of binding sites for $\mathrm{CO}$ in the layer due to this iron doping. For hydrogen $\left(\mathrm{H}_{2}\right)$ no such relation was found but the sensing ability was identical for both layer materials. This observation was related to a different sensing mechanism for $\mathrm{H}_{2}$ which is driven by the diffusion into the layer instead of adsorption on the surface.

Conclusion: The gas sensing selectivity can be enhanced by tuning the properties of the thin film overcoating. A relation of the binding sites in the doped and undoped $\mathrm{SnO}_{\mathrm{x}}$ films and the gas sensing abilities for $\mathrm{CO}$ and $\mathrm{C}_{3} \mathrm{H}_{8}$ was found. This could open the path for optimized gas sensing devices with different coated SPREE sensors. 


\section{Introduction}

Gas sensors are an important tool for example in the fields of process monitoring, workplace safety or environmental analysis. Due to the wide field of possible applications, many different concepts for gas sensing were developed in the past. Typical state-of-the-art concepts for gas measurements are electrochemical and infrared sensors, pellistors and metal oxide conductive sensors (MOS) [1]. All sensing concepts have their respective properties. Electrochemical sensors are limited to several gas species [2]. The infrared (IR) sensor needs a certain optical path for the IR beam inside the gas volume but shows high sensitivity and selectivity [3]. Pellistors can only measure specific gases due to their catalytic combustion concept but are fast and accurate [4]. Conductive MOS sensors show sensitivities to the ppb range but have high cross sensitivities to other gases [5] These sensors detect gases by measuring resistance changes due to the adsorption of gas molecules on the surface $[6,7]$. This surface enhanced and adsorption driven concept has a potential for a wide range of applications because it depends on the gas-surface interaction. This dependence enables us to tune the adsorption properties for specific gases by using different preparation techniques or introduce doped thin films. In the last years, the use of tin oxide $\left(\mathrm{SnO}_{\mathrm{x}}\right)$ layers for gas sensors has attracted some interest [8-10]. These layers are widely used due to their excellent performance in the detection of gases. Due to the high dependency of the sensitivity of these layers in the gas detection on the preparation procedure, several different coating methods were developed which include chemical vapor deposition [11], sol-gel [12], spray pyrolysis [13], sputtering [14-16] and electron beam evaporation [17]. In our approach, we aim to develop a new sensing concept which combines the adsorption concept of MOS sensors with optical ellipsometric readout from the backside which means that the probing laser light does not need a pathway inside the gas volume. Additionally, the cross sensing problems can be solved by modifications enhancing the sensing probability for only one gas species. The aim of this study is a deeper understanding of the relation of the overcoating tin oxide layers and their gas sensing abilities.

\section{Methods \\ Experimental}

The sensors were prepared by a combination of two different techniques which were already reported $[15,16]$. The uncoated rectangular substrate prisms are made of N-BK7 glass with a leg length of $7 \mathrm{~mm}$ (Edmund Optics). The prisms were coated on the hypotenuse face with a $45 \mathrm{~nm}$ gold layer by using the electron beam evaporation technique (CS 730 ECS, von Ardenne Anlagentechnik $\mathrm{GmbH}$ ). The additional undoped $\mathrm{SnO}_{\mathrm{x}}$ add-on layer was then added by using radio frequency (RF) magnetron sputtering (CS 730 ECS, von Ardenne Anlagen- technik $\mathrm{GmbH}$ ) with $13.56 \mathrm{MHz}$ frequency and $200 \mathrm{~W}$ power. Here, a commercially available pure $\mathrm{SnO}_{2}$ target (99.9\%) obtained from FHR GmbH was used. The target has a diameter of $200 \mathrm{~mm}$ and a thickness of $6 \mathrm{~mm}$. For the Fe-doped samples, a home-built RF magnetron sputtering equipment with 13.56 MHz frequency was used with a $\mathrm{SnO}_{2}$ target (99.95\%) of $48 \mathrm{~mm}$ diameter. In this case, an additional rectangular Fe-strip (99.95\%) of size $10 \mathrm{~mm} \times 20 \mathrm{~mm}$ and a thickness of $2 \mathrm{~mm}$ was attached at the center of the $\mathrm{SnO}_{2}$ target. The deposition was done by applying $50 \mathrm{~W}$ of DC pulsed power at a frequency of $50 \mathrm{kHz}$ with a pulse duration of $4 \mu \mathrm{s}$. Depending on the intended layer properties, the parameters of the sputtering were changed. These parameters are the partial pressure of argon and (if added) oxygen, bias and deposition time. For the undoped $\mathrm{SnO}_{\mathrm{x}}$ layers, pure argon with a pressure of $0.3 \mathrm{~Pa}$ was used. The Fe-doped $\mathrm{SnO}_{\mathrm{x}}$ was deposited with a $20 \% / 80 \%$ oxygen/argon mixture with a pressure of $0.2 \mathrm{~Pa}$. The thickness of the $\mathrm{SnO}_{\mathrm{x}}$ and $\mathrm{Fe}: \mathrm{SnO}_{\mathrm{x}}$ overcoatings was $7 \mathrm{~nm}$ and $5 \mathrm{~nm}$, respectively. The thickness was determined by using spectroscopic ellipsometry. The layer morphology was also determined by means of atomic force microscopy (AFM) and transimission electron mircoscopy (TEM) measurements. These measurements are reported in $[18,19]$. It was found that the intended layer structure (glass-gold- $\mathrm{SnO}_{\mathrm{x}}$ ) was achieved and the surface roughness is low (root mean square average of height deviation $R_{\mathrm{q}}<0.2 \mathrm{~nm}$ ). Due to the low $R_{\mathrm{q}}$ value, roughness effects were not taken into account for explanation of the results. Furthermore, no indication of changes in the porosity of the layers which probably could effect the gas sensing ability was found.

The gas sensing technique called surface plasmon resonance enhanced ellipsometry (SPREE) has been described previously [20-22]. The scheme of the device is shown in Figure 1. It consists of the sensing unit with the prepared layer system which is placed onto a gas tube which was prepared with a hole. The gas tube is filled with a gas mixture consisting of synthetic air $\left(20 \% \mathrm{O}_{2}, 80 \% \mathrm{~N}_{2}\right)$ and one added analyte gas $\left(\mathrm{CO}, \mathrm{H}_{2}\right.$ or $\mathrm{C}_{3} \mathrm{H}_{8}$ ). The analyte gas was mixed with synthetic air by using a home-built mixing setup which is regulated by a MKS $647 \mathrm{C}$ multi gas controller monitor. The flow was kept constant at $100 \mathrm{sccm}$. The pressure inside the gas tube was manipulated by using a high-precision valve (SS-6BMG-MM, Swagelok). The unit of sensor and attached gas tube is placed into the measurement spot of a single wavelength laser ellipsometer (Sentech SE 400). The ellipsometer is equipped with a helium-neon laser source $(632.8 \mathrm{~nm})$, a polarizer $(\mathrm{P})$, a switchable prism compensator $(C)$ and a rotating analyzer $\left(A_{R}\right)$. The angle of incidence (AOI) can be changed by means of a manual goniometer with a precision of $0.05^{\circ}$. 


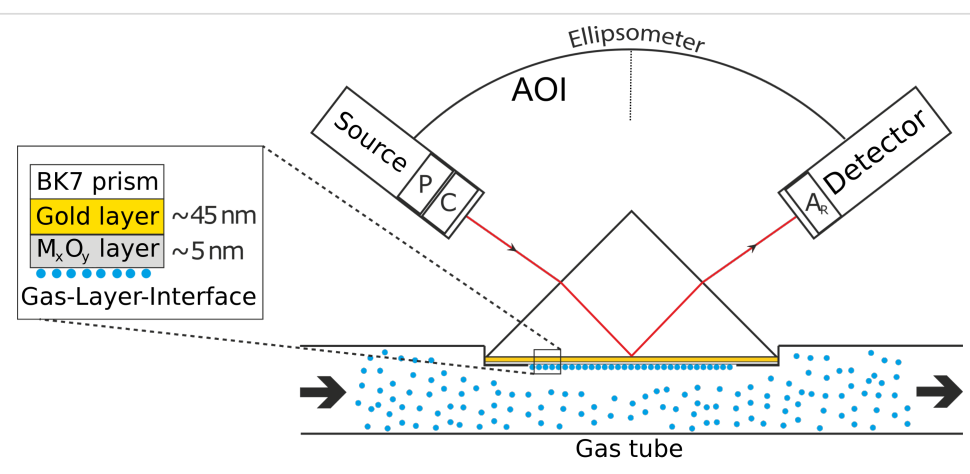

Figure 1: Scheme of the SPREE gas sensing device.

\section{Surface plasmon resonance enhanced ellipsometry}

A theoretical description of the SPREE technique is given in detail elsewhere [23-25]. In ellipsometry, the complex reflectance ratio $\rho$ after irradiation of a sample with linear polarized light is measured. For a simple two-media dielectric interface, the complex reflectance ratio is given by $[26,27]$

$$
\rho=\frac{r_{\mathrm{p}}}{r_{\mathrm{s}}}=\frac{\left|r_{\mathrm{p}}\right| \exp \left(i \delta_{\mathrm{p}}\right)}{\left|r_{\mathrm{s}}\right| \exp \left(i \delta_{\mathrm{s}}\right)}=\tan \Psi \exp (i \Delta),
$$

with $r_{\mathrm{p}, \mathrm{s}}$ as the Fresnel coefficent and $\delta_{\mathrm{p}, \mathrm{s}}$ as the phase for the $\mathrm{p}$ and s-polarization state. $\tan \Psi$ and $\Delta$ are the consecutive amplitude ratio and phase difference of $\rho$. If a thin semitransparent layer like a gold layer is introduced between the two dielectric media, the overall reflection coefficient will change to

$$
R=\frac{r_{01}+r_{12} \exp (-2 \beta)}{1+r_{01} r_{12} \exp (-2 \beta)}
$$

with $r_{01}$ as the Fresnel coefficient at the interface of the incident layer (BK7 glass) and the thin gold and $r_{12}$ as the interface between the gold layer and the dielectric on the bottom side of the setup (air). The $\beta$ value describes the film phase thickness given by the formula

$$
\beta=\frac{2 \pi}{\lambda} N_{1} \cos \Phi_{1}
$$

with $N_{1}$ as the complex refractive index of medium 1 und $\Phi_{1}$ as the incident light angle in medium 1.

By knowing that the intermediate layer is gold, the effect of the surface plasmon resonance has to be taken into account for the description of the reflection coefficients. Because this phenomenon effects mostly the reflection coefficient of the p-polarized

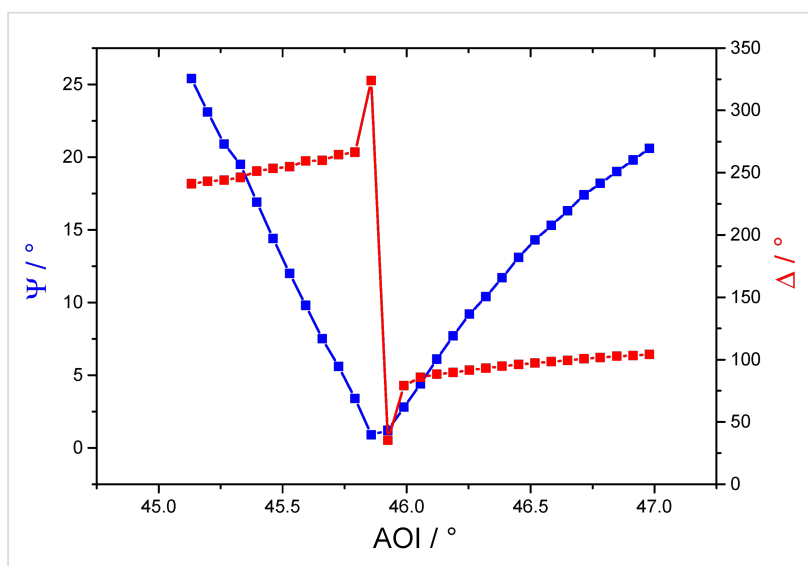

Figure 2: Measured data of $\Psi$ and $\Delta$ for a $B K 7$ glass prism with a $45 \mathrm{~nm}$ Au coating and a $5 \mathrm{~nm} \mathrm{SnO}_{x}$ add-on layer.

part $R_{\mathrm{p}}$, the SPR effect can be described by the reflectance $\mathfrak{R}_{\mathrm{p}}=\left|R_{\mathrm{p}}\right|^{2}$. Close to the surface plasmon resonance, the reflectance can be written as

$$
\mathfrak{R}_{\mathrm{p}}=\left|r_{01}\right|_{\mathrm{p}}^{2}\left[\frac{4 \Gamma^{\infty} \Gamma^{\mathrm{rad}}}{\left(k_{x 0}-\left(k_{\mathrm{SP}}^{\infty^{\prime}}+\Delta k_{\mathrm{SP}}^{\prime}\right)\right)^{2}+\left(\Gamma^{\infty}+\Gamma^{\mathrm{rad}}\right)^{2}}\right]
$$

with $k_{x 0}$ as the component of the wave vector of the incident light in the direction of the surface plasmon propagation and $k_{S P}=k_{\mathrm{SP}}^{\infty^{\prime}}+i \Gamma^{\infty}$ as the wave vector of a surface plasmon on an interface of two semi-infinite media according to [28]. Due to the finite thickness of the gold film, a perturbation term is introduced by using $\Delta k_{\mathrm{SP}}^{\prime}+i \Gamma^{\mathrm{rad}}$. In the ellipsometric measurement the $\Psi$ and $\Delta$ values can be interpreted by [23-25,28].

$$
\tan \Psi=\frac{\left|r_{01}\right|}{\left|r_{\mathrm{s}}\right|} \sqrt{1-\frac{4 \Gamma^{\infty} \Gamma^{\mathrm{rad}}}{\left(k_{x 0}-\left(k_{\mathrm{SP}}^{\infty}+\Delta k_{\mathrm{SP}}^{\prime}\right)\right)^{2}+\left(\Gamma^{\infty}+\Gamma^{\mathrm{rad}}\right)^{2}}}
$$




$$
\begin{aligned}
\Delta & =\arg \frac{\left|r_{01_{\mathrm{p}}}\right|}{\left|r_{\mathrm{s}}\right|} \\
& +\arctan \frac{2\left(k_{x 0}-\left(k_{\mathrm{SP}}^{\infty^{\prime}}+\Delta k_{\mathrm{SP}}^{\prime}\right)\right) \Gamma^{\mathrm{rad}}}{\left(k_{x 0}-\left(k_{\mathrm{SP}}^{\infty^{\prime}}+\Delta k_{\mathrm{SP}}^{\prime}\right)\right)^{2}+\left(\Gamma^{\infty}+\Gamma^{\mathrm{rad}}\right)\left(\Gamma^{\infty}-\Gamma^{\mathrm{rad}}\right)} .
\end{aligned}
$$

If the angle of incidence is varied close to the resonance condition, the $\Psi$ function shows a minimum at the exact resonance angle, while in the $\Delta$ graph a step function can be observed. Both values reach their minimum due to the complete coupling to the plasmon resonance if an ideal system is assumed. If an additional layer is introduced between the gold layer and the ambient dielectric medium (air), the simulation of the system becomes more complex. In this case an extra pertubation has to be taken into account which will disturb the ideal resonance conditions. This case is shown in Figure 2, where a $5 \mathrm{~nm}$ thick tin oxide layer was added to the system. This perturbation leads to a shift of both functions to a higher resonance angle as well as an increased minimum of $\Psi$ and $\Delta$.

\section{Application of the SPR theory to gas sensing}

By changing the dielectric medium (air) with an addition of a certain volume of another gas, a shift in the dependence of $\Psi$ and $\Delta$ on the AOI is predicted. The reason of this change can be explainend by different effects. First, a change of the refractive index of the dielectric medium (air) due to addition of another gas can be considered. In this case, the new refractive index of the gas mixture is given by the Lorenz-Lorentz-equation [29]

$$
\frac{n^{2}-1}{n^{2}+2}=\sum_{i} \rho_{i} \frac{4}{3} \pi \frac{N_{\mathrm{A}}}{M_{i}} \alpha_{i}
$$

with $\rho_{i}$ as the partial density of the $i$ th component, $N_{\mathrm{A}}$ as the Avogadros constant, $M_{i}$ as the molecular weight and $\alpha_{i}$ as the polarizability. By using the changed refractive index of the gas mixture, the values of $\Psi$ and $\Delta$ in the SPREE experiment can be calculated using Equation 5 and Equation 6. However, as the partial density of the added gas is in the dimension of $10^{-6}$ (ppm), this theory does not explain the measured changes in $\Psi$ and $\Delta$ as presented in this study because the quantitative change would be immeasurably small. To explain this sensitivity, the adsorption of molecules on the surface of the sensor has to be taken into account. In this case, as known from SPR spectroscopy [30,31], a new thin layer of adsorbed molecules with the dimension of a few nanometer thickness will change the position of the surface plasmon resonance peak in the angular spectrum to higher angles. This effect depends on the thickness and the refractive index of the layer of adsorbed molecules. In the case of a gas mixture with one gas component added, the thickness of the adsorbed layer is adjusted in correlation with the concentration of this gas in the mixture. If the amount of adsorbed molecules at the surface can be increased, e.g., by providing additional binding sites using a doped overcoating or by enhancing the porosity of the layer, the sensitivity of the gas measurement will be raised. Additionally, the pressure of the gas volume will have an effect to the measured signal because it is proportional to the amount of adsorbed molecules on the surface and because the refracive index of the gas phase rises with the rising pressure.

\section{Results and Discussion Pressure dependence}

As described above, the gas experiments show a pressure dependence, which changes the $\Delta$ response. By changing the relative pressure inside the gas tube, a signal could be detected with the SPREE experiment. The resulting signal in dependence to the relative pressure is shown in Figure 3. The response was measured with steps of 0.1 bar. The pressure was measured by using a gauge relative to atmospheric pressure.

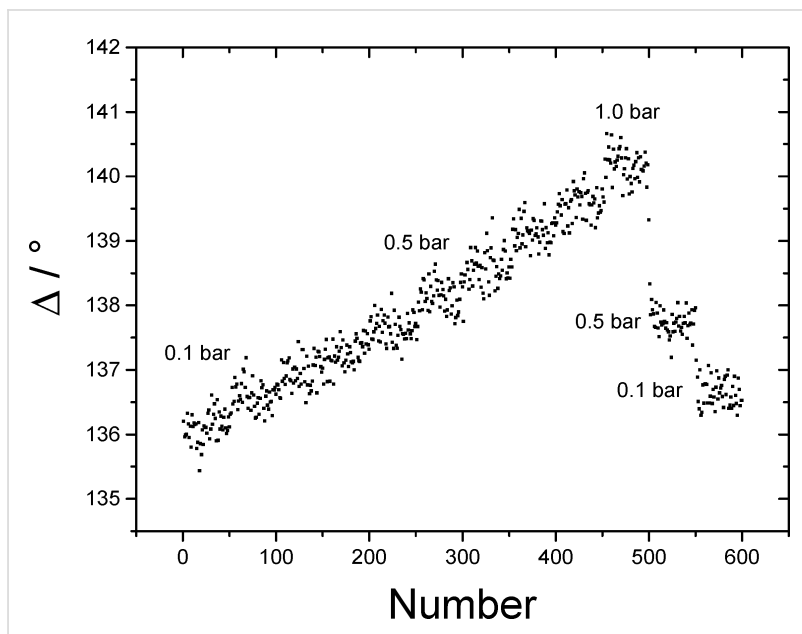

Figure 3: $\Delta$ signal depending on different air pressure values at an $\mathrm{AOI}$ of $47.9^{\circ}$. The pressure was changed every 50 measurement points by 0.1 bar up to a pressure of 1.0 bar. Then the pressure was reduced to 0.5 and 0.1 bar.

This pressure dependence can be a result of the changed amount of molecules adsorbed to the surface which effect the position of the SPR signal. Another explanation could be a changed refractive index of the gas medium due to the different density by increasing the pressure. This behavior shows a linear dependency in the studied pressure region to the pressure which is in agreement with both explanations. Supposedly, a combination of both mechanisms occurs when the total pressure of the gas phase is changed. To achieve the linearity and sensitivity shown in the following gas measurement section, it is necessary to 
keep the pressure constant during the experiment. Otherwise this additional degree of freedom will result in unpredictable response of the $\Delta$ signal to changes in the gas tube. We solved this by using a high precision pressure valve which is adjusted in dependence of the pressure gauge to keep the pressure constant. The pressure value for all gas experiments was adjusted to be 0.4 bar overpressure compared to ambient.

\section{Gas sensing experiments}

A typical gas sensing experiment is shown in Figure 4 where the gas was changed from synthetic air to a mixture of $1000 \mathrm{ppm} \mathrm{CO}$ in synthetic air.
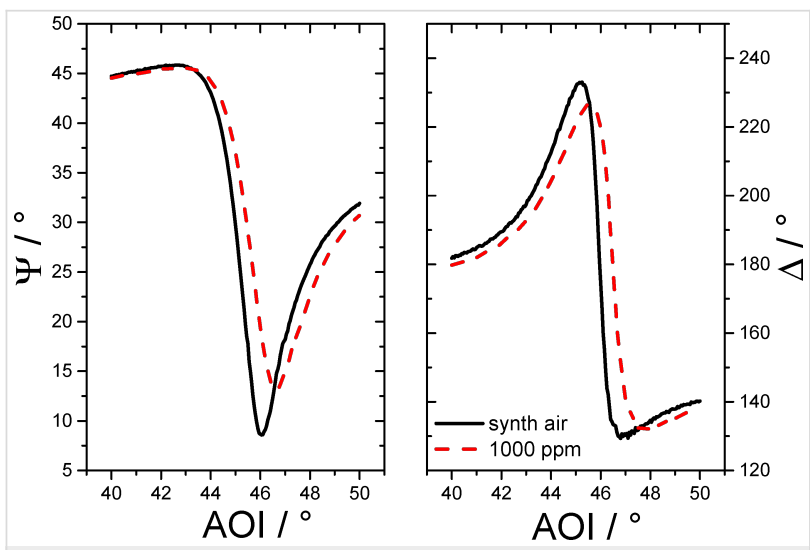

Figure 4: Changes in the $\Psi$ and $\Delta$ angle spectrum due to changing gas atmosphere. Black / solid: synthetic air. Red/dashed 1000 ppm CO in synthetic air.

Here, a shift of $0.5^{\circ}$ of the $\Psi$ minimum and the $\Delta$ phase change can be observed on the AOI axis. The amount of this shift is related to the change in frequency of the surface plasmon resonance due to the adsorption of the gas molecules on the surface as described above. If the response of the $\Psi$ and $\Delta$ signal is monitored over a certain time while the gas concentration is changed, a difference of the signal is measured as shown in Figure 5. The $\Delta \Delta$ value (same for $\Delta \Psi$ ) shows the change of the ellipsometric $\Delta$ value after exposure with a analyte gas concentration in relation to the situation without gas exposure $(\Delta \Delta=0)$. This nomenclature was chosen because only the change of the ellipsometric quantities is relevant for the gas measurement.

We decided to focus on the evaluation of the $\Delta \Delta$ values instead of analyzing the $\Delta \Psi$ values because the signal to noise ratio is much better for the $\Delta \Delta$ measurement. The calculated values of the standard deviation $\sigma$ for the gas measurement of $\mathrm{CO}$ on a $\mathrm{Fe}: \mathrm{SnO}_{\mathrm{x}}$ overlayer, as shown in Figure 5, are $0.04^{\circ}$ for $\Delta \Psi$ and $0.016^{\circ}$ for $\Delta \Delta$, respectively. This lower $\sigma$ value leads to a better signal to noise ratio and, consequently, a higher sensitivity in the gas measurement. The measurement of three different gases

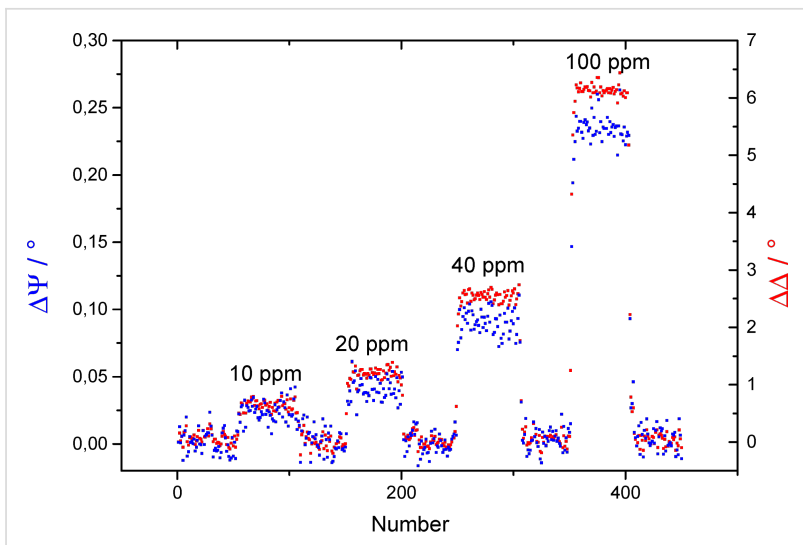

Figure 5: Comparison of the change in $\Psi$ and $\Delta$ at an $\mathrm{AOI}$ of $44.5^{\circ}$ after exposure for different concentrations of $\mathrm{CO}$ on a $\mathrm{Fe}: \mathrm{SnO}_{\mathrm{x}}$ coating. The standard deviations $\sigma$ for $\Delta \Psi$ and $\Delta \Delta$ are $0.04^{\circ}$ and $0.016^{\circ}$, respectively.

is shown in Figure 6 and Figure 7 with $\Delta \Delta$ as the difference in $\Delta$ over the total number of measurements. Each of them represents a ellipsometric measurement and takes approximately $3 \mathrm{~s}$.

It was found that all three gases, $\mathrm{C}_{3} \mathrm{H}_{8}, \mathrm{CO}$ and $\mathrm{H}_{2}$, can be measured down to the low ppm range with different sensitivity. All gases show a fast response to concentration changes. It takes approximately 3-4 measurements to reach the plateau of the signal which represents $9-12 \mathrm{~s}$ response time. This time is likely to be dominated by the time needed for the gas mixture to establish in the apparatus. Therefore, we suppose that all surface processes generating the measurement effect are faster than the time resolution of the experiment. All changes are fully reversible and the signal regenerates to the initial value within the same time of 9-12 s. As shown in Figure 6 and Figure 7, the signal shows different intensities depending on the gas species and layer type. For the undoped $\mathrm{SnO}_{\mathrm{x}}$ add-on layer shown in Figure $6, \mathrm{C}_{3} \mathrm{H}_{8}$ shows the highest signal of $20^{\circ}$ in $\Delta \Delta$ for $100 \mathrm{ppm}$ and is well resolved at $10 \mathrm{ppm}$ with $2.2^{\circ}$. For $\mathrm{H}_{2}$ the maximum signal for $100 \mathrm{ppm}$ reaches $\Delta \Delta=15^{\circ}$ and can only barely identified at $10 \mathrm{ppm}$ with $\Delta \Delta=1.5^{\circ}$. The lowest response was detected for $\mathrm{CO}$ with $\Delta \Delta=7.5^{\circ}$ at $100 \mathrm{ppm}$ and almost no visible signal at $10 \mathrm{ppm}$.

All signals respond linearly to concentration changes as plotted on the right graph in Figure 6. This linearity is a very important aspect of this method for future applications. The different sensitivities to the three measured gases can be explained by the different adsorption behavior of each gas on the surface. By using an undoped $\mathrm{SnO}_{\mathrm{x}}$ layer as the overcoating for the gold sensing layer, the adsorption probability of the sensor layer system is changed. The undoped $\mathrm{SnO}_{\mathrm{x}}$ coating causes a stronger response of the sensor signal for $\mathrm{C}_{3} \mathrm{H}_{8}$ as for $\mathrm{CO}$. This observation supports the assumption that $\mathrm{C}_{3} \mathrm{H}_{8}$ is more likely adsorbed 

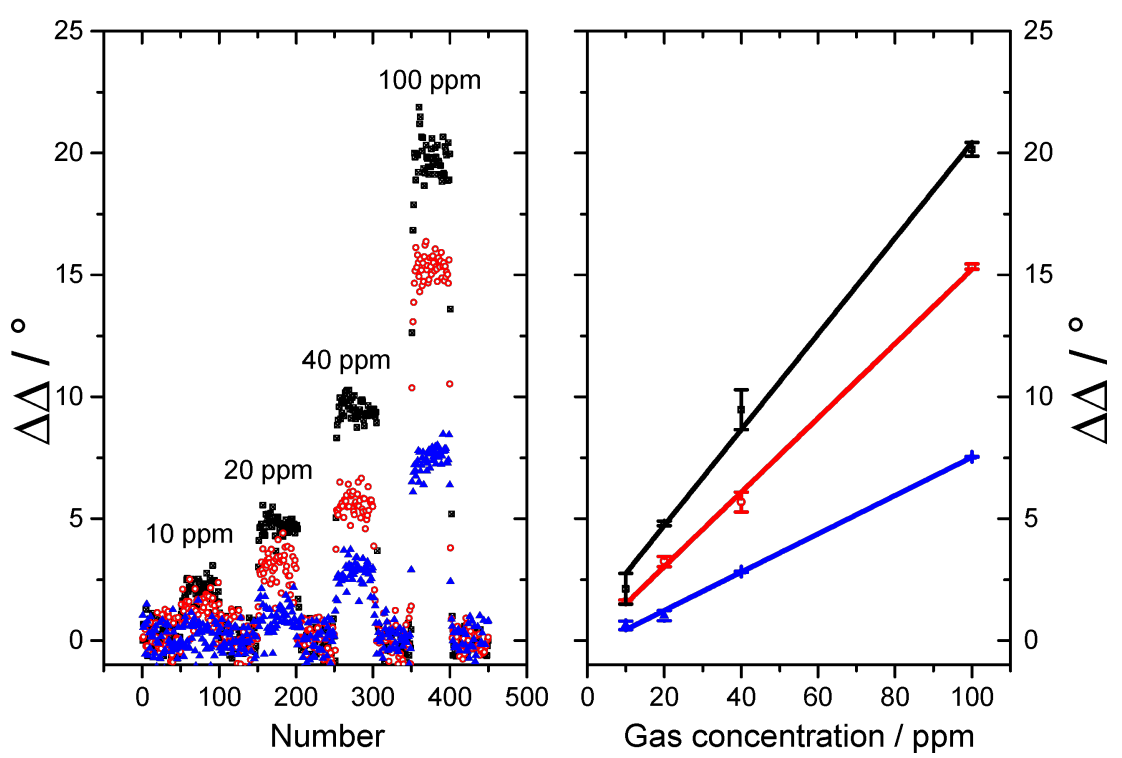

Figure 6: Gas measurement of $\mathrm{C}_{3} \mathrm{H}_{8}$ (black rectangle), $\mathrm{CO}$ (blue triangle) and $\mathrm{H}_{2}$ (red dots) with SPREE with an undoped $\mathrm{SnO} \mathrm{O}_{\mathrm{x}}$ layer at an $\mathrm{AOI}$ of $45.3^{\circ}$ (left). Linearity analysis of the $\Delta \Delta$ response to the gas concentration (right).
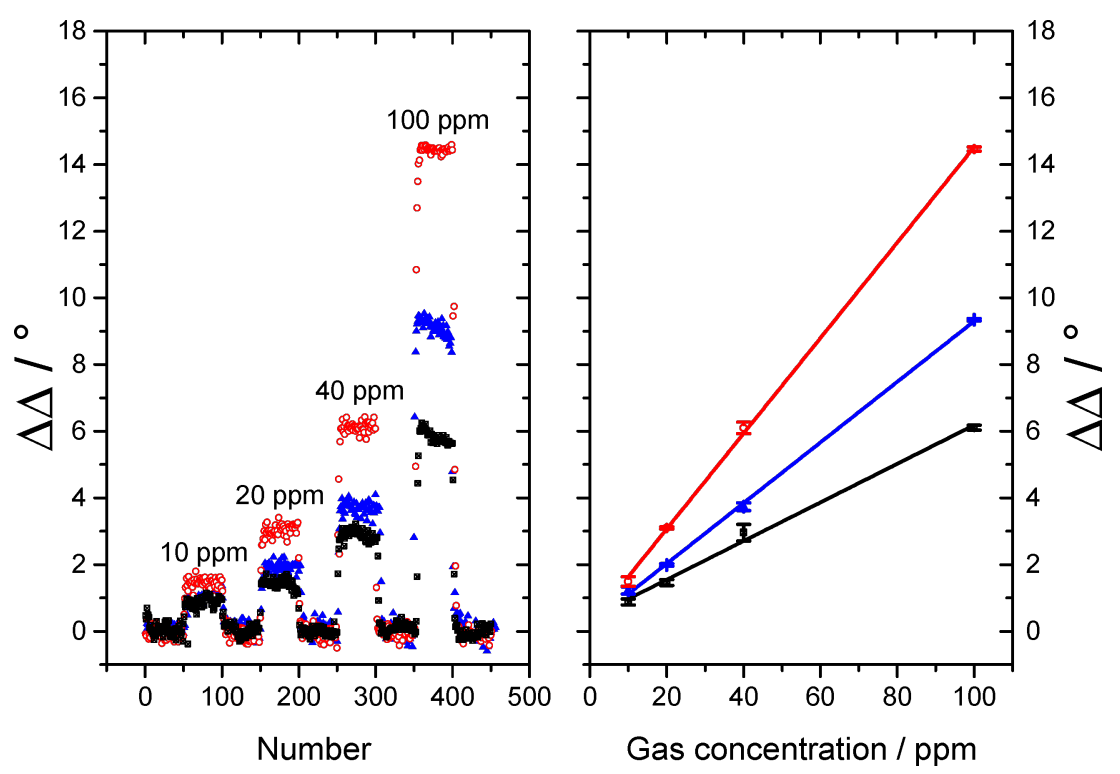

Figure 7: Gas measurement of $\mathrm{C}_{3} \mathrm{H}_{8}$ (black rectangle), $\mathrm{CO}$ (blue triangle) and $\mathrm{H}_{2}$ (red dots) with SPREE at an $\mathrm{AOI}$ of $44.5^{\circ}$ with an Fe:SnO layer (left). Linearity analysis of the $\Delta \Delta$ response to the gas concentration (right).

than CO.In contrast, the response to $\mathrm{H}_{2}$ is driven by an increased diffusion into the sensing layer in contrast to an adsorption at the surface due to the small molecule size [32]. To support the theory of a dependence of the binding sites to the response in the gas measurement, iron doped tin oxide $\left(\mathrm{Fe}: \mathrm{SnO}_{\mathrm{x}}\right)$ overcoated sensors with an increased number of binding sites for $\mathrm{CO}$ were built [33]. This was achieved by adding a block of metallic iron to the target. A gas sensing measurement using a SPR setup with this coating is shown in
Figure 7. By using this type of coating, the response to the gas species and the overall sensitivity has changed. The best response was achieved for $\mathrm{H}_{2}$ with $14.5^{\circ}$ for $100 \mathrm{ppm}$ which is comparable with the response of the undoped $\mathrm{SnO}_{\mathrm{x}}$ layer analyzed in the prior experiment. As the response of $\mathrm{H}_{2}$ is the same for undoped and $\mathrm{Fe}$-doped $\mathrm{SnO}_{\mathrm{x}}$, the assumption that the sensing mechanism is not based on adsorption of the molecule on the surface but on a diffusion process into the sensing layer is supported for this gas species. The signal for $10 \mathrm{ppm}$ is still 
well resolved with $\Delta \Delta=1.6^{\circ}$. The better resolution in comparison to the undoped sample is a result of the much better signal to noise ratio achieved with the $\mathrm{Fe}: \mathrm{SnO}_{\mathrm{x}}$ add-on layer. This effect is probably due to a changed thickness $\left(5 \mathrm{~nm}\right.$ for $\mathrm{Fe}: \mathrm{SnO}_{\mathrm{x}}$ and $7 \mathrm{~nm}$ for $\mathrm{SnO}_{\mathrm{x}}$ ) of the layer which also effects the distinct sharp form of the SPR signal. In another study, we have found that the thickness of the overlayer affects the gas sensing sensitivity but not the selectivity to a specific gas species. By tuning the thickness to a certain limit (depending on the metal oxide system), the ability to measure a lower concentration of the added gas species is improved [18]. The second best response was achieved for CO gas with a value of $\Delta \Delta=9.5^{\circ}$ for $100 \mathrm{ppm}$ and a measurable resolution at $10 \mathrm{ppm}$ with $\Delta \Delta=1.2^{\circ}$. The response is slightly better than in the prior experiment which was ascribed to the higher amount of adsorbed molecules due to increased number of binding sites in the add-on layer. The lowest signal was determined for $\mathrm{C}_{3} \mathrm{H}_{8}$ gas with $\Delta \Delta=6.1^{\circ}$ at $100 \mathrm{ppm}$ and $\Delta \Delta=1.0^{\circ}$ at $10 \mathrm{ppm}$. This extensive decrease in the response for $\mathrm{C}_{3} \mathrm{H}_{8}$ in comparison to the undoped $\mathrm{SnO}_{\mathrm{x}}$ coating corroborates the theory of a reduction of the amount of adsorbed molecules due the raised number of binding sites for CO.

\section{Conclusion}

The recently developed gas measurement technique, surface plasmon resonance enhanced ellipsometry (SPREE), was investigated in terms of sensitivity, reversibility and linearity. Additionally, the relation between changes of the dielectric function due to changes of the gas species and concentration was investigated. It was found that the gas measurement with all probed gas species $\left(\mathrm{H}_{2}, \mathrm{CO}\right.$ and $\left.\mathrm{C}_{3} \mathrm{H}_{8}\right)$ is fully reversible even after several cycles. Furthermore, a linear response to the concentration of every gas species was found which shows a promising behavior for future applications. Further investigations to optimize the sensitivity for specific gas species have shown that the response of the ellipsometric $\Delta$ signal depends on the overcoating of the actual gold sensing layer. Two different add-on layers were tested, undoped $\mathrm{SnO}_{\mathrm{x}}$ and iron doped $\mathrm{SnO}_{\mathrm{x}}$ $\left(\mathrm{Fe}: \mathrm{SnO}_{\mathrm{x}}\right)$ due to their different amount of binding sites for $\mathrm{C}_{3} \mathrm{H}_{8}$ and $\mathrm{CO}$. The assumed effect that an additional amount of binding sites for $\mathrm{CO}$, as present in $\mathrm{Fe}: \mathrm{SnO}_{\mathrm{x}}$, will lead to an increased sensing ability for $\mathrm{CO}$ is supported by the results. In consequence, a decreased signal for $\mathrm{C}_{3} \mathrm{H}_{8}$ was found. No effect in dependence of the doped layer was observed for $\mathrm{H}_{2}$. This was explained by a different sensing mechanism which does not rely on the adsorption of the molecules on the surface but on the diffusion into the sensing layer. However, the detailed sensing mechanism could not be determined due to the lack of temperature dependent measurements. An additional linear dependence to the gas pressure was observed which needs to be controlled during the gas measurement to obtain reliable data on the de- pendence of the measurement signal on the additional layer. The new type of gas sensing with the SPREE method showed promising results by confirming that the sensitivity to a specific gas can be controlled by changing the add-on coating of the sensing gold layer. Additional studies investigating the influence of the add-on layer properties to the gas sensitivity and selectivity will follow.

\section{Acknowledgements}

The authors acknowledge the assistance provided by the Research Infrastructure NanoEnviCz, supported by the Ministry of Education, Youth and Sports of the Czech Republic under Project No. LM2015073 and the funding of Deutsche Forschungsgemeinschaft (DFG-HE4505/3-1). We gratefully thank Andreas Krause for his help with the SPREE setup and Matthias Weise for the layer preparation as well as Dr. Ilona Dörfel and Dr. Werner Österle for the TEM measurements.

\section{References}

1. Arshak, K.; Moore, E.; Lyons, G. M.; Harris, J.; Clifford, S. Sens. Rev. 2004, 24, 181-198. doi:10.1108/02602280410525977

2. Bakker, E.; Telting-Diaz, M. Anal. Chem. 2002, 74, 2781-2800. doi:10.1021/ac0202278

3. Hodgkinson, J.; Tatam, R. P. Meas. Sci. Technol. 2013, 24, 1-59. doi:10.1088/0957-0233/24/1/012004

4. Liu, Y.; Parisi, J.; Sun, X.; Lei, Y. J. Mater. Chem. A 2014, 2, 9919-9943. doi:10.1039/c3ta15008a

5. Barsan, N.; Koziej, D.; Weimar, U. Sens. Actuators, B 2007, 121, 18-35. doi:10.1016/j.snb.2006.09.047

6. McAleer, J. F.; Moseley, P. T.; Norris, J. O. W.; Williams, D. E.; Taylor, P.; Tofield, B. C. Mater. Chem. Phys. 1987, 17, 577-583. doi:10.1016/0254-0584(87)90017-4

7. Becker, T.; Ahlers, S.; Bosch-von Braunmühl, C.; Müller, G.; Kiesewetter, O. Sens. Actuators, B 2001, 77, 55-61. doi:10.1016/s0925-4005(01)00672-4

8. Preiß, E. M.; Rogge, T.; Krauß, A.; Seidel, H. Sens. Actuators, B 2016, 236, 865-873. doi:10.1016/j.snb.2016.02.105

9. Sun, Y.-F.; Liu, S.-B.; Meng, F.-L.; Liu, J.-Y.; Jin, Z.; Kong, L.-T.; Liu, J.-H. Sensors 2012, 12, 2610-2631. doi:10.3390/s120302610

10. Das, S.; Jayaraman, V. Prog. Mater. Sci. 2014, 66, 112-255. doi:10.1016/j.pmatsci.2014.06.003

11. Ray, S. C.; Karanjai, M. K.; Dasgupta, D. Thin Solid Films 1997, 307, 221-227. doi:10.1016/s0040-6090(97)00268-x

12. Jin, Z.; Zhou, H.-J.; Jin, Z.-L.; Savinell, R. F.; Liu, C.-C. Sens. Actuators, B 1998, 52, 188-194. doi:10.1016/s0925-4005(98)00272-x

13. Briand, D.; Labeau, M.; Currie, J. F.; Delabouglise, G. Sens. Actuators, B 1998, 48, 395-402. doi:10.1016/s0925-4005(98)00102-6

14. Shishkin, N. Y.; Zharsky, I. M.; Lugin, V. G.; Zarapin, V. G. Sens. Actuators, B 1998, 48, 403-408. doi:10.1016/s0925-4005(98)00104-x

15. Kormunda, M.; Pavlik, J. Vacuum 2011, 85, 871-874. doi:10.1016/j.vacuum.2010.12.013 
16. Kormunda, M.; Fischer, D.; Hertwig, A.; Beck, U.; Sebik, M.; Pavlik, J.; Esser, N. Thin Solid Films 2015, 595, 200-208.

doi:10.1016/j.tsf.2015.11.009

17. Das, D.; Banerjee, R. Thin Solid Films 1987, 147, 321-331. doi:10.1016/0040-6090(87)90028-9

18. Fischer, D.; Hertwig, A.; Beck, U.; Negendank, D.; Lohse, V.; Kormunda, M.; Esser, N. Appl. Surf. Sci. 2016, in press. doi:10.1016/j.apsusc.2016.11.188

19. Kormunda, M.; Fischer, D.; Hertwig, A.; Beck, U.; Sebik, M.; Esser, N. Phys. Status Solidi A 2016, 213, 2303-2309. doi:10.1002/pssa.201532882

20. Nooke, A.; Beck, U.; Hertwig, A.; Krause, A.; Krüger, H.; Lohse, V.; Negendank, D.; Steinbach, J. Sens. Actuators, B 2010, 149, 194-198. doi:10.1016/j.snb.2010.05.061

21. Nooke, A.; Beck, U.; Hertwig, A.; Krause, A.; Krüger, H.; Lohse, V.; Negendank, D.; Steinbach, J. Thin Solid Films 2011, 519, 2659-2663. doi:10.1016/j.tsf.2010.11.091

22. Beck, U.; Hertwig, A.; Kormunda, M.; Krause, A.; Krüger, H.; Lohse, V.; Nooke, A.; Pavlik, J.; Steinbach, J. Sens. Actuators, B 2011, 160, 609-615. doi:10.1016/j.snb.2011.08.036

23. Arwin, H.; Poksinski, M.; Johansen, K. Appl. Opt. 2004, 43, 3028-3036. doi:10.1364/ao.43.003028

24. Arwin, H. TIRE and SPR-Enhanced SE for Adsorption Processes. Ellipsometry of Functional Organic Surfaces and Films; Springer Series in Surface Sciences, Vol. 52; Springer: Berlin, Germany, 2014; pp 249-264.

25. Oates, T. W. H.; Wormeester, H.; Arwin, H. Prog. Surf. Sci. 2011, 86, 328-376. doi:10.1016/j.progsurf.2011.08.004

26. Azzam, R. M. A.; Bashara, N. M.; Thorburn Burns, D. Anal. Chim. Acta 1987, 199, 283-284. doi:10.1016/S0003-2670(00)82849-4

27. Fujiwara, H. Spectroscopic ellipsometry: principles and applications; John Wiley \& Sons: New York, NY, U.S.A., 2007.

28. Raether, H. Surface plasmons on smooth and rough surfaces and on gratings; Springer: Berlin, Germany, 1988.

29. Owens, J. C. Appl. Opt. 1967, 6, 51-59. doi:10.1364/ao.6.000051

30. Homola, J.; Yee, S. S.; Gauglitz, G. Sens. Actuators, B 1999, 54, 3-15. doi:10.1016/s0925-4005(98)00321-9

31. Li, M.; Cushing, S. K.; Wu, N. Analyst 2015, 140, 386-406. doi:10.1039/c4an01079e

32. Sakai, G.; Baik, N. S.; Miura, N.; Yamazoe, N. Sens. Actuators, B 2001, 77, 116-121. doi:10.1016/s0925-4005(01)00682-7

33. Sung, S. S.; Hoffmann, R. J. Am. Chem. Soc. 1985, 107, 578-584. doi:10.1021/ja00289a009

\section{License and Terms}

This is an Open Access article under the terms of the Creative Commons Attribution License

(http://creativecommons.org/licenses/by/4.0), which permits unrestricted use, distribution, and reproduction in any medium, provided the original work is properly cited.

The license is subject to the Beilstein Journal of Nanotechnology terms and conditions: (http://www.beilstein-journals.org/bjnano)

The definitive version of this article is the electronic one which can be found at:

doi:10.3762/bjnano. 8.56 